\title{
Postoperative air leak grading is useful to predict prolonged air leak after pulmonary lobectomy
}

Sang Gi Oh${ }^{1}$, Yochun Jung ${ }^{1 *+}$, Sanghoon Jheon ${ }^{2}$, Yunhee $\mathrm{Choi}^{3}$, Ju Sik Yun ${ }^{4}$, Kook Joo Na ${ }^{4}$ and Byoung Hee Ahn ${ }^{1 * \dagger}$

\begin{abstract}
Background: Results of studies to predict prolonged air leak (PAL; air leak longer than 5 days) after pulmonary lobectomy have been inconsistent and are of limited use. We developed a new scale representing the amount of early postoperative air leak and determined its correlation with air leak duration and its potential as a predictor of PAL.

Methods: We grade postoperative air leak using a 5-grade scale. All 779 lobectomies from January 2005 to December 2009 with available medical records were reviewed retrospectively. We devised six 'SUM' variables using air leak grades in the initial $72 \mathrm{~h}$ postoperatively.

Results: Excluding unrecorded cases and postoperative broncho-pleural fistulas, there were 720 lobectomies. PAL occurred in 135 cases (18.8\%). Correlation analyses showed each SUM variable highly correlated with air leak duration, and the SUM $\mathrm{Mtog}_{\text {, }}$ which was the sum of six consecutive values of air leak grades for every $8 \mathrm{~h}$ record on postoperative days 2 and 3, was proved to be the most powerful predictor of PAL; PAL could be predicted with $75.7 \%$ and $77.7 \%$ positive and negative predictive value, respectively, when $S_{U} M_{4 \text { to9 }} \geq 16$. When 4 predictors derived from multivariable logistic regression of perioperative variables were combined with SUM $\mathrm{Stog}_{4}$ there was no significant increase in predictability compared with SUM 4 tog alone.

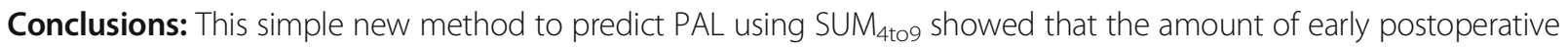
air leak is the most powerful predictor of PAL, therefore, grading air leak after pulmonary lobectomy is a useful method to predict PAL.
\end{abstract}

Keywords: Prolonged air leak, Lobectomy, Air leak grade

\section{Background}

There is no clear consensus on the duration of prolonged air leak (PAL) [1], which is usually considered as lasting longer than 5 or 7 days postoperatively. Air leaking after pulmonary resection is natural, but its prolongation increases the risks of other pulmonary complications such as empyema [2] and unnecessarily lengthens hospital stay [3, 4]. Therefore, accurate prediction of PAL could enable early, selective postoperative

\footnotetext{
* Correspondence: yochuni@naver.com; bhahn@chonnam.ac.kr

tEqual contributors

${ }^{1}$ Department of Thoracic and Cardiovascular Surgery, Chonnam National University Hospital, Chonnam National University School of Medicine, 42 Jebong-ro, 501-757 Dong-gu, Gwangju, South Korea

Full list of author information is available at the end of the article
}

management to prevent PAL possible, which in turn help to reduce the complication risks and hospital costs.

Many studies to elucidate the risk factors of PAL have been made to predict its occurrence [5-10], but the results were inconsistent and therefore, of limited use clinically. Thus, rather than identifying the risk factors, we sought to determine whether observing the pattern of postoperative air leak might be a more direct and accurate way, based on a simple assumption: 'the larger, the longer'.

In this study, the authors developed a new quantitative scale to express the amount of early postoperative air leak to determine its correlation with air leak duration and possibility as a predictor of PAL. 


\section{Methods}

The medical records of 779 lobectomies conducted consecutively at our institution from January 2005 to December 2009 were reviewed retrospectively. This study was approved by Seoul National University Bundang Hospital's Institutional Review Board, and the need for informed consent was waived (IRB number: B-1010-113-103).

\section{Grading of air leak and definition of time period ' $P$ '}

We began implementing a chest tube management protocol including quantitative assessment of air leak using a 5-grade scale (Table 1) and were able to evaluate almost all postoperative air leaks since December 2004. After pulmonary resection, in our hospital, air leak grades are recorded on the vital sheet along with blood pressure or heart rate. Air leak grading, based on evaluation of volitional coughing by the patient, was performed by specially trained general nurses and recorded every $8 \mathrm{~h}$, because of their 3 shifts per day. Therefore, to facilitate postoperative data collection, the authors defined one 8-h period corresponding to one nursing shift as a time scale one ' $\mathrm{P}$ ' and expressed every postoperative 8-h period as P1, P2, P3, etc. (Fig. 1).

\section{Definition of air leak cessation and PAL}

We generally do not remove the chest tube until the drainage amount decreases to less than $150-200 \mathrm{~mL}$ within the previous $24 \mathrm{~h}$. Therefore, chest tube removal time cannot reflect the time when air leak stops, so prior to performing this study, air leak cessation was necessary to be defined clearly based on the grading records. Air leak cessation was defined as air leak grade 0 or 1 being continued for 3 Ps $(24 \mathrm{~h})$. In cases with 2 chest tubes, air leak cessation was timed based on the late-ceased one. In this study, PAL was defined as air leak persisting more than 5 days (15 Ps).

\section{'SUM' variables as predictors of PAL}

For quantitative comparison of early postoperative air leak, we devised 6 'SUM' variables, based on which we evaluated the degree of air leak from P1 to P9 (Table 2).

Table 1 Air leak grading

\begin{tabular}{ll}
\hline Grade & Definition \\
\hline 0 & No air bubble on three serial volitional coughs \\
1 & More than one air bubbles on three \\
& serial volitional coughs \\
2 & Persistent air bubbles on volitional coughs \\
3 & Persistent, small amount of air bubbles \\
& on spontaneous respiration \\
4 & Persistent, large amount of air bubbles \\
& on spontaneous respiration \\
\hline
\end{tabular}

In cases having 2 chest tubes, the larger one among the two values of air leak grade in the same $\mathrm{P}$ was selected for evaluation. Six SUM variables representing the amount of early postoperative air leak were examined respectively to see whether they correlated with air leak duration or not. Then, among the variables proved to correlate with air leak duration, the most optimal variable and its cutoff value for prediction of PAL were obtained. Other predictors for PAL derived from preoperative and intraoperative variables of this study cohort were also tested as to whether they had any additive effect on optimizing prediction of PAL.

\section{Validation of the reliability of air leak grading}

For 1 week while conducting this study, we had one or two pairs of nurses from each shift assess the air leak grade simultaneously but independently, and one of the authors (Jung Y) compared the two grades to monitor the agreement on air leak grading.

\section{Statistical analysis}

Spearman rank correlation coefficients were calculated to investigate the correlation between each SUM variable and air leak duration. Receiver operating characteristic (ROC) curve analyses were performed and areas under the ROC curves (AUC) were compared to select the best predictive SUM variable, then its cutoff value that optimized both sensitivity and specificity was obtained.

The distribution of preoperative and intraoperative variables were compared between PAL (+) and PAL (-). Categorical variables were expressed as the frequency and percentage of cases and compared by either the $x^{2}$ test or Fisher's exact test, as necessary. For a continuous variable with a nonnormalized distribution, data were reported as a median with range and compared by the Mann-Whitney U-test. The significant factors determined by a two-tailed nominal $P$ value of $<0.2$ in univariable analysis were entered into a multivariable logistic regression to identify predictors of PAL. Stepwise logistic regression was performed to control multicollinearity among predictors and for each element remaining in the multivariable model, a $P$ value, odds ratio (OR), and 95\% confidence interval (CI) were calculated. The Hosmer-Lemeshow goodness-of-fit statistic was used to evaluate the model fit. The resultant predictors and the optimal SUM variable were combined to create a multivariable logistic regression, the AUC of which was compared with that of the optimal SUM variable regarding predictive ability [11]. A two-sided P value of $<0.05$ was considered to indicate a statistically significant difference. In order to assess the agreement on air leak grading, the weighted kappa statistic was performed. The analysis was carried out using PASW 


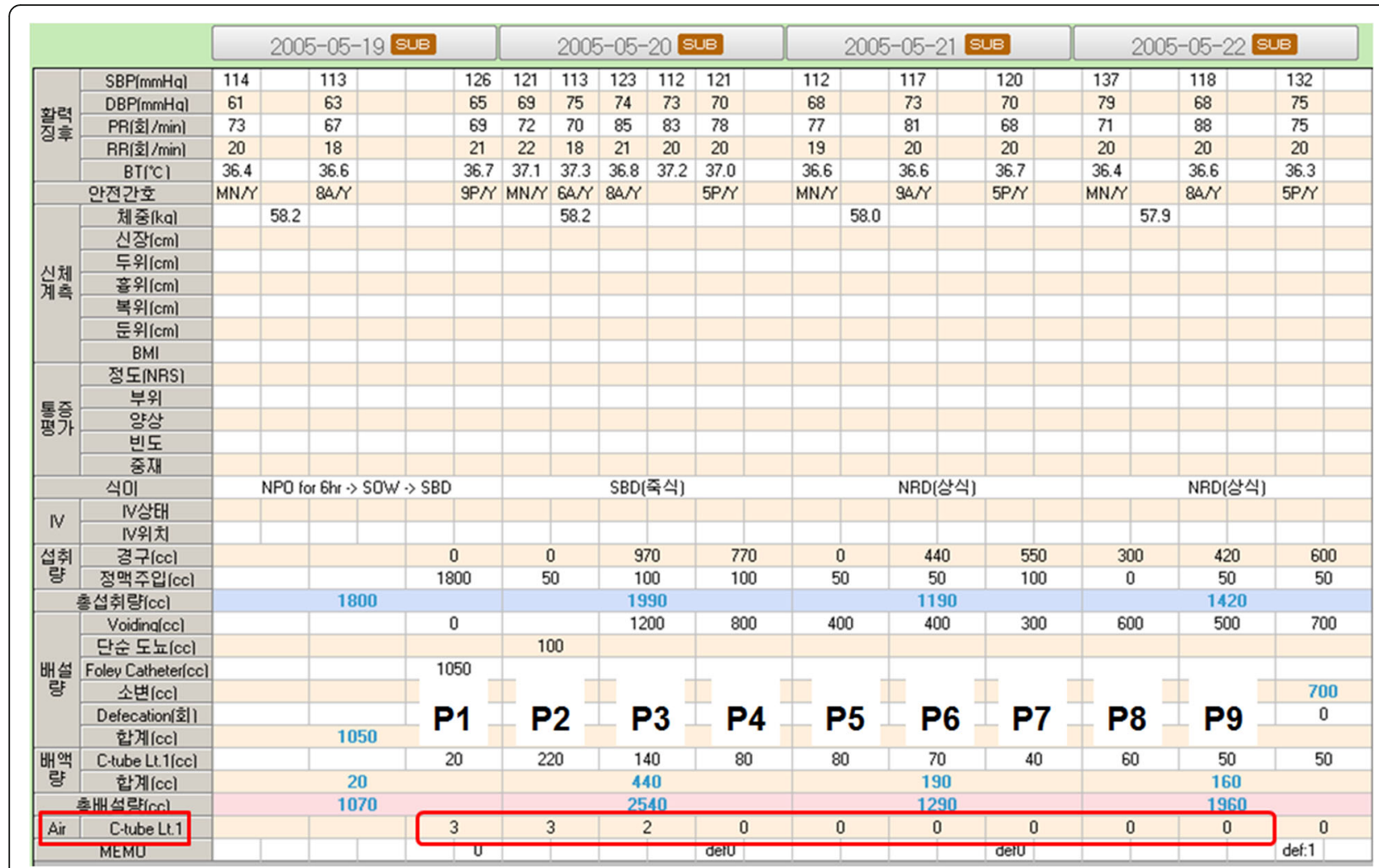

Fig. 1 Actual air leak grading chart in our hospital. Air leak grades were recorded every $8 \mathrm{~h}$ (each " $\mathrm{P}$ ") postoperatively. In this case, air leak ceased at $\mathrm{P} 6$ and the $\mathrm{SUM}_{4 \text { tog }}$ was 0

Statistics, version 17.0 (SPSS Inc.; Chicago, IL) and SAS, version 9.1 (SAS Institute; Cary, NC).

\section{Results}

Excluding the cases of 53 lacking graded records, 3 having 3 chest tubes, 2 postoperative broncho-pleural fistulas, and 1 followed by sequential operation in a situation where air leak after initial lobectomy had not ceased, a total of 720 consecutive lobectomies were included in this study. The most common preoperative diagnosis was malignant lung neoplasm (including pulmonary metastasis) occupying $89.9 \%$. The number of cases with single chest tube was $372(51.7 \%)$. Other characteristics of this study cohort are summarized in Table 3.

Median duration of postoperative air leak was 2.7 (0.758.0) days and the median of chest tube removal was at postoperative day (POD) 4.7 (0.7-67.0), and that of discharge was POD 7 (2-187). The numbers of cases with persistent air leak at P4, P7, P10, P13 and P16 are 577, $410,275,184$, and 127, respectively. A total of 127 cases with persistent air leak at P16 were considered as PAL and 8 other cases were also regarded as PAL despite their

Table 2 Definitions of 6 'SUM' variables

\begin{tabular}{|c|c|c|c|c|c|c|c|c|c|}
\hline Postoperative period & P1 & $\mathrm{P} 2$ & P3 & P4 & P5 & P6 & P7 & P8 & P9 \\
\hline Air leak grade ${ }^{a}$ & $0-4$ (N1) & $0-4$ (N2) & $0-4(N 3)$ & $0-4(\mathrm{~N} 4)$ & $0-4$ (N5) & $0-4$ (N6) & $0-4$ (N7) & $0-4$ (N8) & $0-4(N 9)$ \\
\hline Variable & \multicolumn{9}{|c|}{ Definition } \\
\hline $\mathrm{SUM}_{1 \text { to3 }}$ & \multicolumn{9}{|c|}{$\mathrm{N} 1+\mathrm{N} 2+\mathrm{N} 3$} \\
\hline $\mathrm{SUM}_{4 \mathrm{to}}$ & \multicolumn{9}{|c|}{$\mathrm{N} 4+\mathrm{N} 5+\mathrm{N} 6$} \\
\hline $\mathrm{SUM}_{1 \text { to6 }}$ & \multicolumn{9}{|c|}{$\mathrm{N} 1+\mathrm{N} 2+\mathrm{N} 3+\mathrm{N} 4+\mathrm{N} 5+\mathrm{N} 6$} \\
\hline $\mathrm{SUM}_{7 \text { to9 }}$ & \multicolumn{9}{|c|}{$\mathrm{N} 7+\mathrm{N} 8+\mathrm{N} 9$} \\
\hline $\mathrm{SUM}_{4 \mathrm{to} 9}$ & \multicolumn{9}{|c|}{$\mathrm{N} 4+\mathrm{N} 5+\mathrm{N} 6+\mathrm{N} 7+\mathrm{N} 8+\mathrm{N} 9$} \\
\hline $\mathrm{SUM}_{1 \text { to9 }}$ & \multicolumn{9}{|c|}{$\mathrm{N} 1+\mathrm{N} 2+\mathrm{N} 3+\mathrm{N} 4+\mathrm{N} 5+\mathrm{N} 6+\mathrm{N} 7+\mathrm{N} 8+\mathrm{N} 9$} \\
\hline
\end{tabular}

${ }^{\mathrm{a}} \mathrm{N}$ ' represents the air leak grade, from 0 to 4 , for the given postoperative period ' $\mathrm{P}$ ' 
Table 3 Baseline characteristics and results of univariable analyses

\begin{tabular}{|c|c|c|c|c|}
\hline \multirow[t]{3}{*}{ Variables } & \multicolumn{3}{|c|}{ No. of cases (\%) } & \multirow[t]{3}{*}{$P$ value } \\
\hline & All & PAL (-) & $\operatorname{PAL}(+)$ & \\
\hline & $(N=720)$ & $(N=585)$ & $(N=135)$ & \\
\hline Age $^{\mathrm{b}}$ (years) & $64(7-85)$ & $64(7-85)$ & $66(14-82)$ & $<0.001^{c}$ \\
\hline Male & $464(64.4 \%)$ & $354(60.5 \%)$ & $110(81.5 \%)$ & $<0.001$ \\
\hline \multicolumn{5}{|l|}{ Preoperative medication } \\
\hline Aspirin or NSAIDs & $98(13.6 \%)$ & $80(13.7 \%)$ & $18(13.3 \%)$ & 0.92 \\
\hline Steroid (including inhalator) & $15(2.1 \%)$ & $9(1.5 \%)$ & $6(4.4 \%)$ & $0.05^{d}$ \\
\hline Neoadjuvant chemotherapy & $50(6.9 \%)$ & $39(6.7 \%)$ & $11(8.1 \%)$ & 0.54 \\
\hline \multicolumn{5}{|l|}{ Preoperative spirometry } \\
\hline $\mathrm{FEV}_{1} / \mathrm{FVC}<70 \%$ & $259(36.0 \%)$ & $201(34.4 \%)$ & $58(43.0 \%)$ & 0.04 \\
\hline FVC\% $<80$ & $76(10.6 \%)$ & $62(10.6 \%)$ & $14(10.4 \%)$ & 0.90 \\
\hline $\mathrm{FEV}_{1} \%<80$ & $108(15.0 \%)$ & $81(13.8 \%)$ & $27(20.0 \%)$ & 0.04 \\
\hline $\mathrm{D}_{\mathrm{L}} \mathrm{CO} \%<80$ & $103(14.3 \%)$ & $81(13.8 \%)$ & $22(16.3 \%)$ & 0.34 \\
\hline Underlying DM & $101(14.0 \%)$ & $83(14.2 \%)$ & $18(13.3 \%)$ & 0.80 \\
\hline Preoperative $\mathrm{Hb}<10 \mathrm{~g} / \mathrm{dL}$ & $29(4.0 \%)$ & $23(3.9 \%)$ & $6(4.4 \%)$ & $0.41^{d}$ \\
\hline Preoperative albumin $<3.0 \mathrm{~g} / \mathrm{dL}$ & $12(1.7 \%)$ & $10(1.7 \%)$ & $2(1.5 \%)$ & $0.32^{d}$ \\
\hline $\mathrm{BMI}<25.5 \mathrm{~kg} / \mathrm{m}^{2}$ & $523(72.6 \%)$ & $416(71.1 \%)$ & $107(79.3 \%)$ & 0.07 \\
\hline Open thoracotomy & $375(52.1 \%)$ & $299(51.1 \%)$ & $76(56.3 \%)$ & 0.28 \\
\hline Right side operation & $456(63.3 \%)$ & $352(60.2 \%)$ & $104(77.0 \%)$ & $<0.001$ \\
\hline Upper lobectomy & $372(51.7 \%)$ & $293(50.1 \%)$ & $79(58.5 \%)$ & 0.08 \\
\hline Combined chest wall resection & $21(2.9 \%)$ & $17(2.9 \%)$ & $4(3.0 \%)$ & $1.00^{d}$ \\
\hline Pleural adhesion (+) & $338(46.9 \%)$ & $256(43.8 \%)$ & $82(60.7 \%)$ & $<0.001$ \\
\hline Incomplete fissure (+) & $362(50.3 \%)$ & $284(48.5 \%)$ & $78(57.8 \%)$ & 0.05 \\
\hline
\end{tabular}

NSAID denotes non-steroidal anti-inflammatory drug, DM diabetes mellitus, $\mathrm{Hb}$ hemoglobin, $B M I$ body mass index. In spirometry variables, $F E V_{1}$ denotes forced expiratory volume in one second, FVC forced vital capacity, $D_{L} C O$ diffusing capacity of the lung for carbon monoxide. Suffix '\%' means '\% of predicted normal'

${ }^{a} P$ values are for comparison between PAL $(-)$ and PAL $(+)$

bxceptionally, ages are expressed as 'median (range)'

cMann-Whitney U-test was performed

${ }^{d}$ Fisher's exact test was performed. In other cases, $x^{2}$ test was performed

air leak duration being $\leq 15$ Ps, because pleurodesis had been conducted for prophylaxis of PAL, so the total occurrence of PAL in this study cohort was 135 (18.8\%).

\section{The larger, the longer?}

Based on 526 cases having a chest tube beyond POD 3, correlation analyses showed that each SUM variable strongly correlated with air leak duration. The Spearman rank correlation coefficient ranged from the lowest of 0.71 for $\mathrm{SUM}_{1 \text { to3 }}$ to the highest of 0.87 for $\mathrm{SUM}_{4 \text { to9 }}$ (Table 4), which is thought to not only mean that all SUM variables can play a role as predictors of PAL, but also support the basic assumption of this study that the larger the early postoperative air leak, the longer it will persist.

\section{Prediction of PAL by the SUM scale}

PAL occurred in 134 cases (48.7\%) out of 275 cases with persistent air leak at P10. ROC curves were generated for each SUM variable. The calculated AUC revealed that $\mathrm{SUM}_{4 \text { to9 }}$ to be the most powerful predictor of PAL (the largest AUC; 0.819. Table 4), and PAL could be predicted with $76.9 \%$ sensitivity and $76.6 \%$ specificity with $75.7 \%$ positive predictive value and $77.7 \%$ negative predictive value, when $\mathrm{SUM}_{4 \text { to } 9} \geq 16$.

Univariable analysis revealed significant differences between PAL $(+)$ and PAL $(-)$ in regard to age, male gender, preoperative steroid medication, $\mathrm{FEV}_{1} / \mathrm{FVC}<$

Table 4 Statistical values of SUM variables

\begin{tabular}{lcc}
\hline Variables & Spearman rank correlation coefficient & Calculated AUC \\
\hline SUM $_{1 \text { to3 }}$ & 0.71 & 0.71 \\
SUM $_{\text {to6 }}$ & 0.81 & 0.78 \\
SUM $_{\text {7to9 }}$ & 0.83 & 0.81 \\
SUM $_{1 \text { to6 }}$ & 0.81 & 0.77 \\
SUM $_{4 \text { to9 }}$ & $\mathbf{0 . 8 7}$ & $\mathbf{0 . 8 2}$ \\
SUM $_{\text {to9 }}$ & 0.87 & 0.81 \\
\hline
\end{tabular}

AUC denotes area under the ROC curve

The bold data show that SUM4to9 is the most strongly associated parameter with the air leak duration (i.e. the highest Spearman correlation coefficient) and the most powerful predictor of PAL (i.e. the largest AUC) 
$70 \%, \mathrm{FEV}_{1} \%<80, \mathrm{BMI}<25.5 \mathrm{~kg} / \mathrm{m}^{2}$, right side operation, upper lobectomy, presence of pleural adhesion, and incomplete fissure (Table 3). Stepwise logistic regression analysis with each associated variable determined by univariable analysis revealed only male gender, right side operation, presence of pleural adhesion, and $\mathrm{BMI}<$ $25.5 \mathrm{~kg} / \mathrm{m}^{2}$ as significantly associated with PAL (Table 5). When these 4 predictors were combined with $\mathrm{SUM}_{4 \text { to9 }}$ into a multivariable logistic regression model to predict PAL, there was no significant increase in predictability compared with that of $\mathrm{SUM}_{4 \mathrm{to}}$ alone (calculated AUC 0.83 vs. 0.82 respectively, $P=0.46$ ) (Fig. 2).

\section{Reliability of air leak grading}

A total of 33 pairs of air leak grades were collected. Twenty two pairs of ward nurses participated in the grading. There were 7 disagreements on air leak grading, but the degrees of difference were never more than a single grade. The weighted kappa coefficient between the 2 measurements of air leak grade was 0.88 (95\% CI: 0.79-0.96), indicating a very good interobserver agreement [12].

\section{Discussion}

We set out to determine whether our newly developed scale could be useful as a predictor of PAL. Although there have been many studies to predict PAL, most of which have tried to determine predictive risk factors by comparing PAL groups with other groups and reported various risk factors such as poor pulmonary function $[6,8-10]$, poor nutrition [7], or specific operative findings $[8,10]$, but their results were not consistent with each other and therefore are of limited clinical use. Brunelli et al. [10] in 2010, unlike previous studies, reported a risk factor scoring system for prediction of PAL. He elicited 4 predictors from a study group of 658 lobectomy patients: age $>65$ years, presence of pleural adhesion, $\mathrm{FEV}_{1} \%<80$, and $\mathrm{BMI}<25.5 \mathrm{~kg} / \mathrm{m}^{2}$, and different scores were given to the factors according to their weights. The scoring system was validated externally in 233 other hospital patients. However it also had a limitation because of its low positive predictive value of about $25 \%$.

Various investigators have examined the concept that grading the amount of early postoperative air leak might be helpful in predicting PAL [13, 14]. In 2001, Cerfolio

Table 5 Results of multivariable logistic regression

\begin{tabular}{lllcc}
\hline Predictors & OR & $P$ value & & \multicolumn{2}{c}{$95 \% \mathrm{Cl}$} & \\
\cline { 4 - 5 } & & & Lower & Upper \\
\hline Right side operation & 2.37 & $<0.001$ & 1.52 & 3.70 \\
Male & 2.66 & $<0.001$ & 1.65 & 4.28 \\
Pleural adhesion & 1.76 & 0.005 & 1.18 & 2.62 \\
$\mathrm{BMl}<25.5 \mathrm{~kg} / \mathrm{m}^{2}$ & 1.72 & 0.02 & 1.08 & 2.75 \\
\hline
\end{tabular}

OR denotes odds ratio, $\mathrm{Cl}$ confidence interval, $B M \mathrm{l}$ body mass index

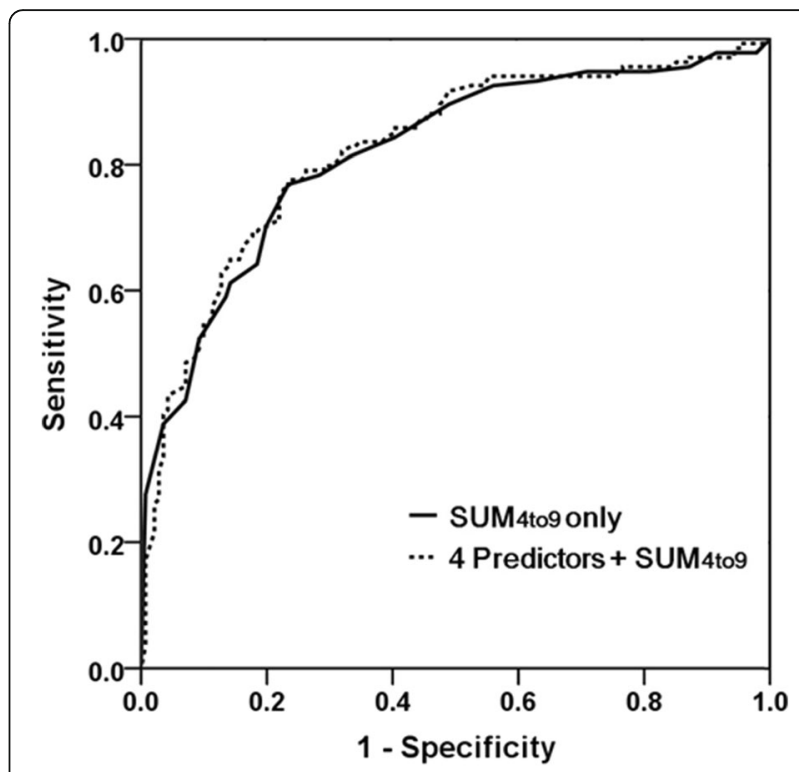

Fig. 2 Comparison of the 2 AUCs. The solid line represents the ROC curve derived from $S_{4} M_{4 t o 9}$ only (AUC 0.82) and the dotted line, combination of 4 perioperative risk factors of PAL and $S_{4} M_{4 \text { tog }}$ (AUC 0.83). There was no statistically significant difference $(P=0.46)$

et al. [13] reported that PAL can be predicted by air leak grade on POD 1. In their study, a commercially available air-leak meter, scoring leaks from 1 to 7 with 7 being the highest, was used. However, originally his work aimed to evaluate the effectiveness of water seal for stopping air leaks. Thus he did not give much weight to air leak grading as a predictor of PAL. In addition, the air leak grading system devised by Cerfolio et al. requires special equipment, and thus the system is not widely used.

To the best of our knowledge, the present study is the first practical attempt to focus only on quantifying air leakage in the early postoperative period to predict PAL. The easy-to-use variable, $\mathrm{SUM}_{4 \text { to9 }}$, has the highest positive predictive value among reports until now. Our air leak grading system, to obtain $\mathrm{SUM}_{4 \text { to9, }}$, needs no special equipment, and yet it is very convenient to apply in the clinical field. Based on our results, we can now decide whether to wait or perform a reintervention (e.g. pleurodesis or redo surgery) for air leak cessation on POD 3.

In addition to proposing a practical and effective method to predict PAL, this study tangibly confirms our hypothesis that the amount of early postoperative air leak predicts air leak duration, by correlation analyses of SUM variables with air leak duration. Furthermore, it reveals that other preoperative or intraoperative variables do not increase the predictive power of $\mathrm{SUM}_{4 \mathrm{to}}$. These findings can be integrated to mean that 1) grading postoperative air leak might be the only factor needed to predict PAL, and 2) the effects of various possible factors contributing to prolongation of air leak might combine 
to result in the grade of early postoperative air leak. Therefore, future studies aiming at providing more accurate prediction of PAL will have to focus on the evaluation of early postoperative air leak in terms of when, by what method, and how frequently it should be measured, not on other indirect factors.

However, this study has the following potential limitations. First of all, the retrospective nature of the study might have incurred some problems in defining and recording the variables. In particular, since air leak cessation was determined retrospectively from medical records, there might have been some discrepancy between the real and the defined cessations. Essentially, our air leak grading is based on subjective assessment, albeit by specially trained nurses, so some might question its reliability. However, over the past several years, we have empirically recognized that its simplicity enables strong interobserver agreement, as shown in the test to see the reliability of our air leak grade. Recently developed digital airflowmetry may be helpful in further increasing interobserver agreement in future studies.

\section{Conclusions}

We developed an easy-to-use method to predict PAL using a new scale, $\mathrm{SUM}_{4 \text { to9 }}$, derived from our own air leak grading protocol. This study proved that the amount of early postoperative air leak is the most powerful predictor of PAL, therefore, grading air leak after pulmonary lobectomy is a useful method to predict PAL.

\section{Abbreviations \\ AUC: Area under the ROC curve; BMI: Body mass index; $\mathrm{D}_{\mathrm{L}} \mathrm{CO} \%$ : \% of predicted normal diffusing capacity of the lung for carbon monoxide; DM: Diabetes mellitus; $F E V_{1} \%$ : \% of predicted normal forced expiratory volume in one second; FVC\%: \% of predicted normal forced vital capacity; Hb: Hemoglobin; NSAID: Non-steroidal anti-inflammatory drug; PAL: Prolonged air leak; POD: Postoperative day; ROC curve: Receiver operating characteristic curve; VATS: Video-assisted thoracoscopic surgery}

\section{Acknowledgements}

The authors give their warmest thanks to Professor J. Patrick Barron of Department of International Communications Center of Tokyo Medical University for his kind pro vono review of this manuscript, and to Professor Cheong Lim of Department of Thoracic and Cardiovascular Surgery, Seoul National University Bundang Hospital, for his editing of the figure. We also appreciate the statistical analysis provided by the Medical Research Collaborating Center at Seoul National University College of Medicine / the Seoul National University Hospital, and thank the DSYU English Editing Service and Editage (www.editage.co.kr) for English language editing.

\section{Funding}

This study received no external funding and was completely supported by the budget of Department of Thoracic and Cardiovascular Surgery, Seoul National University Bundang Hospital.

\section{Availability of data and materials}

The dataset supporting the conclusions of this article is included within the article and its additional file.

\section{Authors' contributions}

YJ and BHA initially proposed the basic idea of air leak grading for prediction of PAL. SGO and YJ designed this study and were principally involved in data collection, statistical analysis, interpretation, and manuscript writing. SJ also participated in designing this study and was involved in the writing of manuscript. YC was principally involved in selecting appropriate statistical method and validating the results. JSY, KJN, and BHA made a significant contribution to this manuscript in the process of initial research design, data analysis, and revision of the draft. All authors read and approved the final manuscript.

\section{Competing interests}

The authors declare that they have no competing interests.

\section{Consent for publication}

Not applicable.

\section{Ethics approval and consent to participate}

This study was approved by Seoul National University Bundang Hospital's Institutional Review Board, with patient consent waived (IRB number: B-1010-113-103).

\section{Author details}

'Department of Thoracic and Cardiovascular Surgery, Chonnam National University Hospital, Chonnam National University School of Medicine, 42 Jebong-ro, 501-757 Dong-gu, Gwangju, South Korea. ${ }^{2}$ Department of Thoracic and Cardiovascular Surgery, Seoul National University Bundang Hospital, Seongnam, South Korea. ${ }^{3}$ Medical Research Collaborating Center, Seoul National University College of Medicine / Seoul National University Hospital, Seoul, South Korea. ${ }^{4}$ Department of Thoracic and Cardiovascular Surgery, Chonnam National University Hwasun Hospital, Chonnam National University School of Medicine, Hwasun, South Korea.

Received: 11 July 2016 Accepted: 19 January 2017

Published online: 23 January 2017

\section{References}

1. Adebonojo SA. How prolonged is "prolonged air leak"? Ann Thorac Surg. 1995;59:549-50.

2. Brunelli A, Xiume F, Al Refai M, Salati M, Marasco R, Sabbatini A. Air leaks after lobectomy increase the risk of empyema but not of cardiopulmonary complications: a case-matched analysis. Chest. 2006:130:1150-6.

3. Bardell T, Petsikas D. What keeps postpulmonary resection patients in hospital? Can Respir J. 2003;10:86-9.

4. Varela G, Jimenez MF, Novoa N, Aranda JL. Estimating hospital costs attributable to prolonged air leak in pulmonary lobectomy. Eur J Cardiothorac Surg. 2005;27:329-33.

5. Singhal S, Ferraris VA, Bridges CR, Clough ER, Mitchell JD, Fernando HC, et al. Management of alveolar air leaks after pulmonary resection. Ann Thorac Surg. 2010;89:1327-35.

6. Abolhoda A, Liu D, Brooks A, Burt M. Prolonged air leak following radical upper lobectomy: an analysis of incidence and possible risk factors. Chest. 1998;113:1507-10.

7. Isowa N, Hasegawa S, Bando T, Wada H. Preoperative risk factors for prolonged air leak following lobectomy or segmentectomy for primary lung cancer. Eur J Cardiothorac Surg. 2002;21:951.

8. Brunelli A, Monteverde M, Borri A, Salati M, Marasco RD, Fianchini A. Predictors of prolonged air leak after pulmonary lobectomy. Ann Thorac Surg. 2004;77:1205-10. discussion 10.

9. Stolz AJ, Schutzner J, Lischke R, Simonek J, Pafko P. Predictors of prolonged air leak following pulmonary lobectomy. Eur J Cardiothorac Surg. 2005;27:334-6.

10. Brunelli A, Varela G, Refai M, Jimenez MF, Pompili C, Sabbatini A, et al. A scoring system to predict the risk of prolonged air leak after lobectomy. Ann Thorac Surg. 2010;90:204-9.

11. DeLong ER, DeLong DM, Clarke-Pearson DL. Comparing the areas under two or more correlated receiver operating characteristic curves: a nonparametric approach. Biometrics. 1988;44:837-45.

12. Altman DG. Practical statistics for medical research. London: Chapman and Hall; 1991. p. 404

13. Cerfolio RJ, Bass C, Katholi CR. Prospective randomized trial compares suction versus water seal for air leaks. Ann Thorac Surg. 2001;71:1613-7.

14. Sakamoto T, Nishio W, Okada M, Harada H, Uchino K, Tsubota N. Management of air leak after pulmonary resection. Jpn J Thorac Cardiovasc Surg. 2004;52:292-5. 